\title{
CONTEXTUALLY EMBODIED AGENTS
}

\author{
Catherine Pelachaud \\ Department of Computer Science, University of Rome "La Sapienza", Rome, Italy
}

Key words: embodied agent, face-to-face communication, communicative facial expression, 3D facial model

\begin{abstract}
In this chapter we present our work on the elaboration of a contextually embodied agent. We define such an agent as an agent capable of planning what to communicate as well as deciding with which verbal and nonverbal signals to output considering contextual information. We first present our 3D facial model that follows MPEG-4 standard. Then we present our taxonomy of communicative facial expressions. We describe our discourse planner that generate synchronized verbal and nonverbal signals. Our planner takes as input a communicative goal and output a multimodal discourse where nonverbal expressions are modulated based on contextual information.
\end{abstract}

\section{INTRODUCTION}

As computers are being more and more part of our world we feel the urgent need of proper user interface to interact with. The use of command lines typed on a keyboard are obsolete, specially as computers are receiving so much attention from a large audience. The metaphor of face-to-face communication applied to human-computer interaction is receiving a lot of attention (André et al., 1998; Cassell, 2000; Poggi et al., 2000; Johnson et al., 2000; Nass et al., 2000). Humans are used since they are born to communicate with others. Seeing faces, interpreting their expression, understanding speech are all part of our development and growth. But faceto-face conversation is very complex phenomenon as it involved a huge number of factors. We speak with our voice, but also with our hand, eye, 
face and body. Our gesture modifies, emphasizes, contradicts what we say by words. The production of speech and nonverbal behaviors work in parallel and not in antithesis. They seem to be two different forms (voice and body gestures) of the same process (speech). Nonverbal behaviors add information on what will be difficult to express by words. They may have the function of an adverb or adjective to modulate what is being said. For example, they can express emotion, attitude toward the others. One cannot imagine the production of one behavior without the occurrence of the other ones. It is therefore important to consider both verbal and nonverbal behaviors while building an embodied agent. The agent must have the capacity to decide which facial expressions to show, which words to say with which intonation. The choice of the communicative act (word, eye movement, facial expression) to perform is based on whom we are taking to, in which context the conversation takes place. Of course this choice is not a necessarily conscious one. We do not talk in the same way to a child, a foreigner, or to an important person. An impulsive person will not express herself in the same manner as a shy person. Our goal is to create a contextually embodied agent, that is an agent capable of planning what to communicate as well as deciding with which verbal and nonverbal signals to output considering contextual information.

In this paper we first present our 3D facial model. We concentrate on the computation of communicative facial expressions and how these expressions are modulated based on contextual information. We will also present our discourse planner that generate embodied verbal and nonverbal signals.

\section{FACIAL MODEL}

Our facial model is based on MPEG-4 standard (Doenges et al., 1997). The model uses a pseudo-muscular approach (Pasquariello, 2000). The muscle contractions are obtained through the deformation of the polygonal network around feature points. Each feature point corresponds to skin muscle attachment. The deformation is performed in a zone of influence that has an ellipsoid shape whose centroid is the feature point. The displacement of points within this area of influence obeys to a deformation function that is function of the distance between the points and the feature point (see figures 1 and 2). 


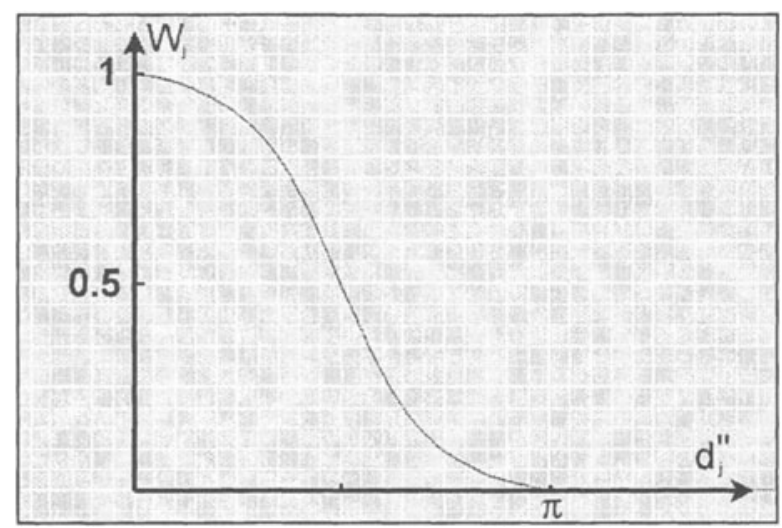

Figure 1: Deformation function

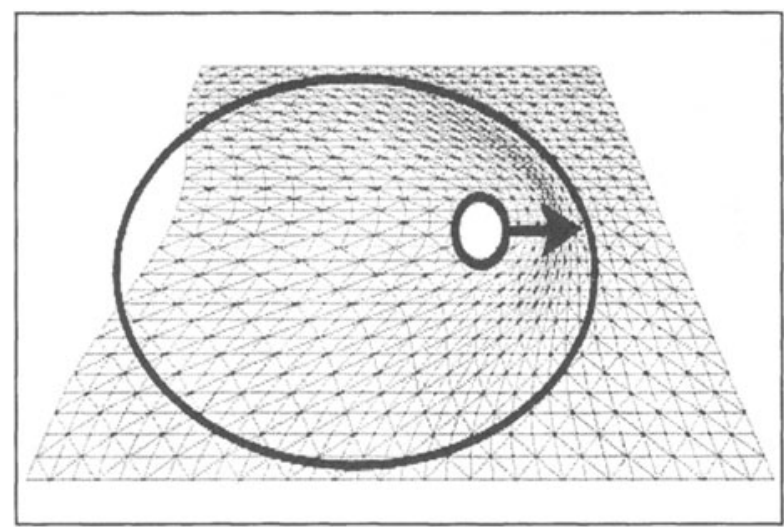

Figure 2: Skin deformation in the area of influence

Two sets of parameters describe and animate the 3D facial model: facial animation parameter set (FAPS) and facial definition parameter (FDP). The FDPs define the shape of the model while the FAPS define the facial actions. When the model has been characterized with FDP, the animation is obtained by specifying for each frame the values of FAPS. The facial model also includes particular features such as wrinkles and furrow to enhance its realism. Brow wrinkles are simulated by discontinuous lines on the forehead and appear when the eyebrows raise (action of the frontalis). They have been implemented using bump mapping technique (see figure 3). Identically frown wrinkles are vertical lines between the two eyebrows and appear under the action of the corrugator. The nasolabial furrow goes from the nose to the corner of the mouth and appears under the action of muscles whose end is around the corner of the mouth. Here bulges and furrows have been modelled using a specialized displacement function that move outward 
points within a specific area. The points of area A that are affected by muscular contraction will be deformed by the muscular displacement function, while the points of area B (area of the bulge / furrow) will be moved outward to simulate the skin accumulation and bulging (see figures 4 , 5 and 6).

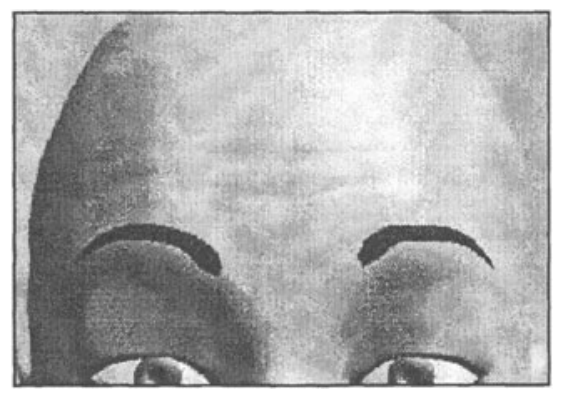

Figure 3: Brow wrinkles

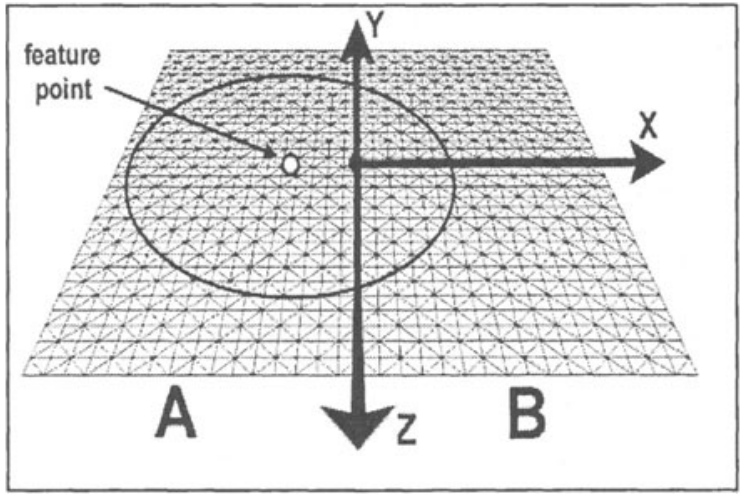

Figure 4:Within the area of influence, the two zones A (muscular traction) and B (accumulation)

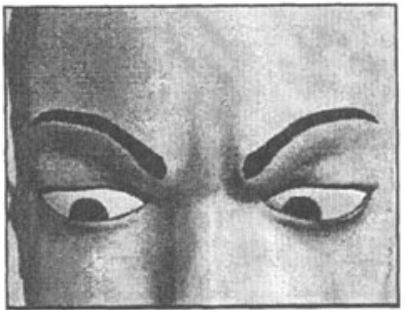

Figure 5: Folds and bulges - frown

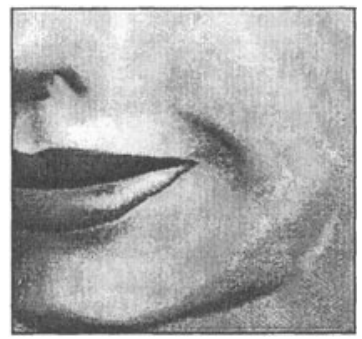

Figure 6: Folds and bulges - smile 


\section{FACIAL COMMUNICATIVE FUNCTIONS}

Faces are an important means of communication and may have several communicative functions. They are used to control the flow of conversation; that is they help in regulating the exchange of speaking turns, keeping the floor or asking for it (Duncan and Fiske, 1985). Actions such as smiling, raising the eyebrows, and wrinkling the nose may accompany the flow of speech and are synchronized at the verbal level, punctuating accented phonemic segments and pauses (Ekman, 1979). Other facial expressions may substitute for a word or string of words, or emphasize what is being said. They can also express attitude toward one own speech (such as irony) or toward the interlocutor (like showing submission). They are also the primary channel to express emotion (Ekman, 1982).

We have decided to consider facial expressions not from the actions involved in the expression (e.g. raising eyebrows) but rather from their communicative functions (Poggi and Pelachaud, 1998). Indeed the same expression may change meaning depending on its place and time of occurrence in the conversation. Raising eyebrows signal surprise but also emphasis of what is being said; they signal question mark, specially in the case of non-syntactically questions but they are also part of the expression used when suggesting something to someone. The expression would vary in their temporal properties (onset and offset values as well as duration of the action) and in the intensity of the actions. Moreover not everybody uses the same expression to carry a given function.

In order to characterize nonverbal communicative functions, we need two information: their meaning and their visual action. The latter ones are described as a list of FAPS parameters that will drive our 3D facial model (see Section 2.). The former ones are represented as a set of goals and beliefs the speaker has the goal to communicate. We therefore differentiate communicative functions in four categories (Poggi et al., 2000):

1. information about speaker's beliefs

2. information about speaker's intentions

3. information about speaker's affective state

4. metacognitive information about speaker's mental state

\subsection{Information about speaker's beliefs}

In this class we gather functions that provide information on speaker's eliefs. The speaker may be certain or uncertain of what she is saying. She may use words like 'may be', 'perhaps', or use conditional such as 'might', 'could' but she can also raise her eyebrow to mark uncertainty. She may 
contrast several elements in her speech, she may precise, restate what she just said... This can be done verbally (e.g., 'because', 'but') but also facially (raising eyebrow to show contrast between beliefs). She may also mimic the property of things (abstract entity or real object): squeezing the eyes when mentioning 'a very subtle nuance between 2 concepts' or a tiny pin on her jacket to imitate how little is the difference or how tiny is the pin. Each function in this class gives information on how certain the speaker is of her belief, how she relates several beliefs with each others and how she believes the property of an object is.

\subsection{Information about speaker's intention}

This class groups several levels of information type.

- single communicative act: It can be about information of the intention of one single communicative act: the performative of a sentence. In a previous work (Poggi and Pelachaud, 1998) we have proposed a formalism to represent performative of a sentence as well as to highlight the existing link between performative verbs and facial expressions. Imploring might be expressed with the eyebrow of sadness while giving an order might be indicated with a frown. There exist three main classes of performative: request, inform and ask. Within a main class there exist several performatives. A request may be suggested, advised, implored. We can warn a person to inform her or we can announce her something. We can interrogate or question her to ask her information. The choice of a particular performative is determined by considering the following elements: a) we will consider consciously or unconsciously the type of social relationship that exists between ourselve and the addressee; $b$ ) we will evaluate how certain or uncertain we are about what we are going to say, and c) we will establish for whom is the action requested. The latest element will distinguish advise vs command in the request class as well as inform vs warn in the inform class. The degree of certainty of our beliefs determines if we will assure our beliefs or we will suggest them. Finally the type of social relationship will decide if we can order the addressee to perform a given action or if we may suggest it. We also found that some facial expressions are linked to performative (Poggi and Pelachaud, 1999). A person $A$ implores a person $B$ to perform an action $a$ as $A$ knows she can not achieve $a$ without the help of $B$. $A$ will be sad if $B$ does not help her. When imploring $A$ will display the expression of sadness (inner eyebrows raised). On the other hand $A$ will raise her eyebrow to mark her uncertainty while suggesting something. 
- whole hierarchy of intentions: Facial expressions are often used to mark new information in a sentence: a raising eyebrow or a head nod and gazing at the addressee usually accompany accented word (Ekman, 1979). These signals emphasize what the speaker makes relevant to the addressee, what she marks as being important in her speech.

- overall arrangement of discourse: A conversation is made of an exchange of speaking turns. During a conversation we may encounter situation where participants start talking simultaneously, or on the opposite no interlocutor takes the speaking floor. One may interrupt speaker's talk to start talking without expecting one's speaking turn. Verbal and nonverbal cues help this process. A turn-taking system (Duncan, 1985) refers to how people negotiate speaking turns in a conversation, or any ritual meeting. In giving the speaking turn, the speaker often gazes at the addressee, her arm and hand come to rest... On the other hand just before taking the speaking turn one gazes at the current speaker and starts gesticulation.

- direct addressee's attention to events: Gaze or head direction may have a deictic function such as pointing gesture. In some situation a gaze may be more useful to indicate a place, person, object... than a gesture. In a social gathering it might be more preferable to refer to the person we are talking about with a simple gaze. It will be less noticeable than a pointing hand...

\subsection{Information about speaker's affective state}

Emotions are best expressed with the face. They may be triggered by an event, action, or a person's action (Ortony et al., 1988). Some emotions are triggered by an event and are not directed toward someone (examples are emotion of fear, surprise). One can feel emotion toward another person (such as love, scorn, hate). Ekman and his colleagues have proposed the existence of universal facial expressions linked to six emotions (Ekman, 1982). Of course for each emotion there exists several variants of these facial expressions. We can bite our lips of anger or show our teeth. Moreover the display of the facial expression of emotion will be modulated by our culture, our social environment, to whom we are talking to... In this case, Ekman refers to Display Rules (Ekman, 1979) that is cultural rules that regulates the display of a given expression. We believe (Decarolis et al., 2000 that other rules regulate the display or non-display of an expression: if we are with a very apprehensive person that we like, we will not show our fear not to frighten her. But if we are a very impulsive person we might not consider this fact and just display our emotion. 


\subsection{Metacognitive information about speaker's mental state}

Metacognitive function provides meta-information on what we are talking about. When we are thinking or remembering something we break our eye gaze with the addressee in order to concentrate on our thoughts. But we can also use such an expression to deliberately show we are thinking. At an oral exam we might ostentatiously display such an expression to make people believe we are deeply thinking.

\section{NOTION OF CONTEXT}

The choice of a communicative act and its display is not part of the speaker's communicative goal from the beginning, but it comes from consideration of context (Poggi and Pelachaud,1998). A speaker decides which specific Performative to use in one's sentence (or other non-verbal communicative act) on the basis of the social situation, the social relationship to the addressee, and of the addressee's cognitive, affective and personality factors. Requesting something to somebody may be performed as an order, a suggestion, an imploration... You will implore somebody if you know that this person has the capacity to get what you want while you can not get it by yourself. On the other hand if you know you have some power over a person you may order him to get what you desire. Each different types of request has an associated facial expression. So the choice of the appropriate signals depends on the situation the conversation takes place and the relation between the speaker and the addressee. Finally the display of a facial expression may be cancelled, masked by another expression, deintensify or intensify depending on the context (Ekman, 1982). If you just pass a difficult exam but your friend did not you will not show too much your happiness not to make him feel worse.

\section{ENRICHED DISCOURSE PLANNER}

As our goal is to have an agent capable of planning what to say and with which signals we have built what we call an enriched discourse planner (Decarolis et al., 2000). The system (see figure 7) takes as input a communicative goal, the goal the agent has to communicate. The Plan library elaborates a first plan where the rhetorical relations between parts of the discourse are specified. This plan is then enriched with information about emotion display. As mentioned in section 3.3, an emotion is triggered by an 
event, action or person. We have implemented C. Elliott's set of rules (Elliott, 1992) that are based on (Ortony et al., 1988) to trigger an emotion.

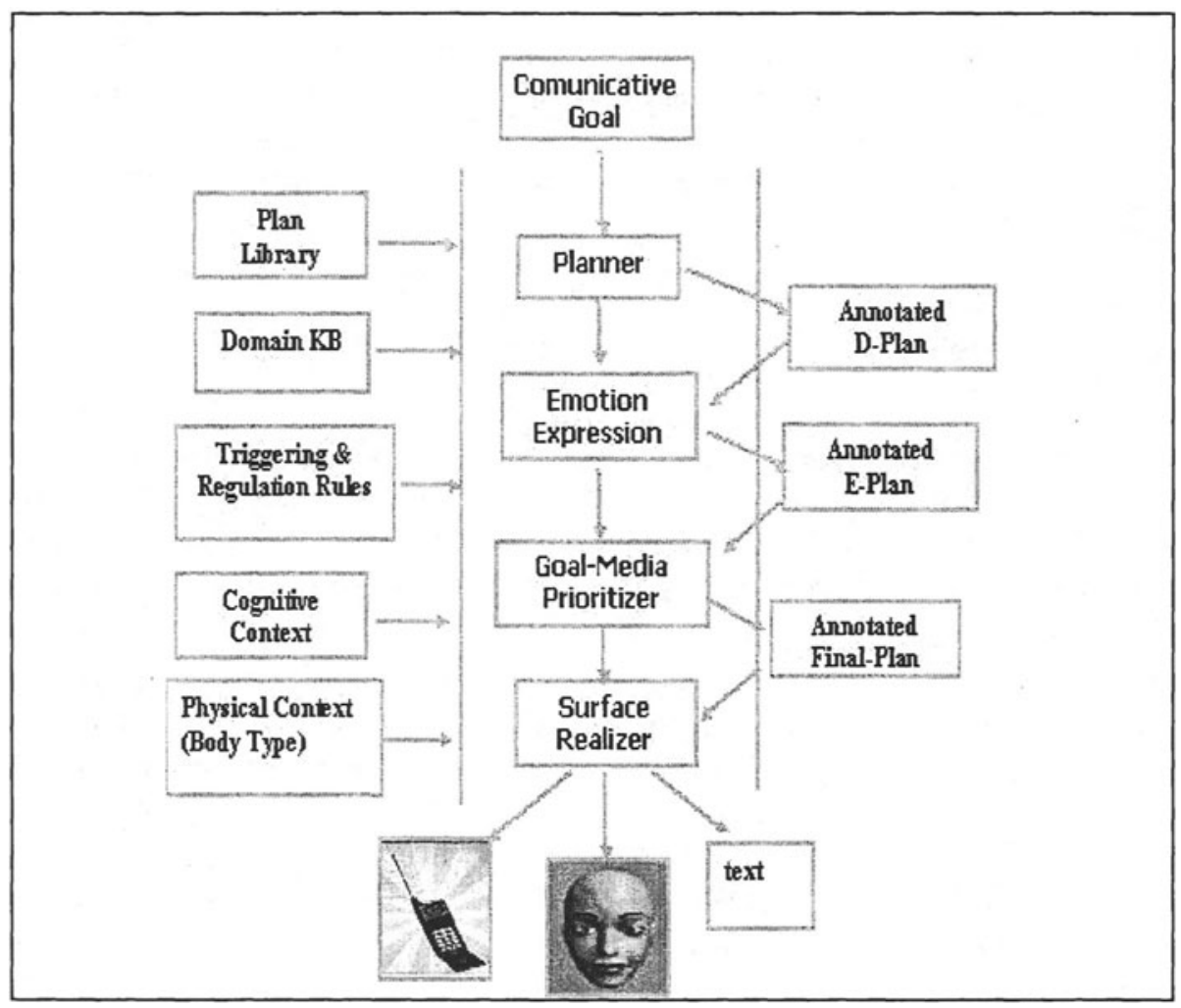

But the display of an emotion is regulated by a set of regulation rules (Decarolis et al., 2000) that are an over set of the display rules introduced by Ekman (1982). These rules take into consideration several elements of the context to decide whether or not an expression should be displayed: personality of the agent and of the addressee, cognitive capacity of the addressee, and relationship between the agent and the addressee. The general structure of the regulation rules is of the type:

IF (Feel Ag e) AND DC-Cond THEN (Display Age)

or

IF (Feel Ag e) AND DC-Cond THEN NOT(Display Ag e) 
Where $A g$ stands for agent, $D C$-Cond for conditions on the context, and $e$ for the given emotion.

Where the regulation rules have been activated, the discourse plan is enriched with these rules. This new plan is then computed as an XML document. For example, such an XML annotated document is:

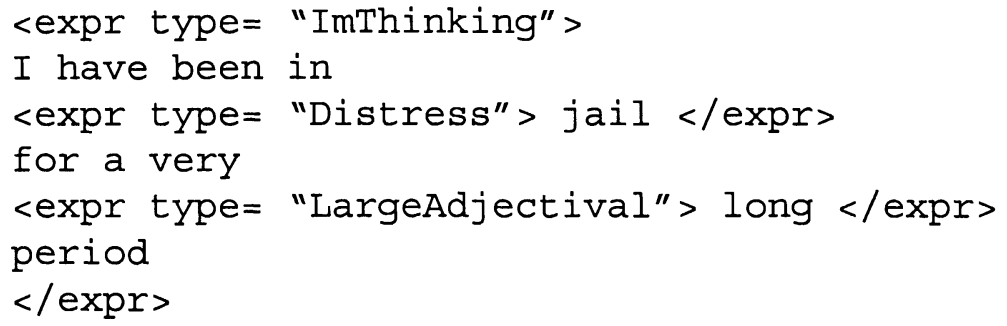

The XML tags are then interpreted and translated into FAPS that drive the $3 \mathrm{D}$ facial model.

\section{CONCLUSION}

In this paper we have presented the architecture underlying the creation of a contextually embodied agent. A taxonomy of nonverbal facial and gaze expressions has been elaborated. This taxonomy is based on the meaning of the communicative behaviors. This allows us to represent the communicative act of a speaker as a set of beliefs and goals and to establish a visual signal (facial behavior, gaze) to each of them. The discourse planner we have implemented has been enriched with triggering and regulation rules as well as with contextual information giving the ability to our agent to retain her expression or not. Our agent may become a reflexive agent not only an impulsive agent!

\section{REFERENCES}

E. André, T. Rist, and J. Mueller. "Integrating reactive and scripted behaviors in a life-like presentation agent." In Proceedings of the second International Conference on Autonomous Agents, pages 261--268, 1998.

J.L. Austin. How to do thinks with words. Oxford University Press, London, 1962.

J. Cassell. "Embodied conversational interface agents." Communications of the ACM, 43(4):70--78, April 2000.

J. Cassell, C. Pelachaud, N.I. Badler, M. Steedman, B. Achorn, T. Becket, B. Douville, S. Prevost, and M. Stone. "Animated conversation: Rule-based generation of facial expression, gesture and spoken intonation for multiple conversational agents." In 
Computer Graphics Proceedings, Annual Conference Series, pages 413--420. ACM SIGGRAPH, 1994.

N. De Carolis, C. Pelachaud, and I. Poggi. " Verbal and nonverbal discourse planning." In Workshop on "Achieving Human-Like Behavior in Interactive Animated Agents workshop", Fourth International Conference on Autonomous Agents, 2000.

P. Doenges, F. Lavagetto, J. Ostermann, I.S. Pandzic, and E. Petajan. "MPEG-4: Audio/video and synthetic graphics/audio for mixed media." Image Communications Journal, 5(4), May 1997.

S. Duncan and D.W. Fiske. Interaction Structure and Strategy. Cambridge University Press, 1985.

P. Ekman. "About brows: Emotional and conversational signals." In M. von Cranach, K. Foppa, W. Lepenies, and D. Ploog, editors, Human ethology: Claims and limits of a new discipline: contributions to the Colloquium, pages 169--248. Cambridge University Press, Cambridge, England; New-York, 1979.

P. Ekman. Emotion in the human face. Cambridge University Press, 1982.

C. Elliott. An Affective Reasoner: A process model of emotions in a multiagent system. $\mathrm{PhD}$ thesis, Northwestern University, The Institute for the Learning Sciences, 1992. Technical Report No. 32.

W.L. Johnson, J.W. Rickel, and J.C. Lester. "Animated pedagogical agents: Face-to-face interaction in interactive learning environments." To appear in International Journal of Artificial Intelligence in Education, 2000.

C. Nass, K. Isbister, and E.J. Lee. "Truth is beauty: Researching embodied conversational agents." In S. Prevost J. Cassell, J. Sullivan and E. Churchill, editors, Embodied Conversational Characters. MITpress, Cambridge, MA, 2000.

A. Ortony, G.L. Clore, and A. Collins. The Cognitive Structure of Emotions. Cambridge University Press, 1988.

S. Pasquariello. Modello per l'animazione facciale in MPEG-4. Master's thesis, University of Rome, 2000.

I. Poggi and C. Pelachaud. Performative faces. Speech Communication, 26:5--21, 1998.

I. Poggi and C. Pelachaud. "Emotional meaning and expression in performative faces." In International Workshop on Affect in Interactions: Towards a New Generation of Interfaces, Annual Conference AC'99 of the EC I3 Programme, Siena, October 1999.

I. Poggi, C. Pelachaud, and F. de Rosis. "Eye communication in a conversational (3D synthetic agent." Special Issue on Behavior Planning for Life-Like Characters and Avatars of AI Communications, 2000. 